\title{
LA INCLUSION EN EDUCACION INFANTIL EN ESPAÑA A PARTIR DE UN \\ ESTUDIO DE CASOS SOBRE LOS ESPACIOS ESCOLARES Y SU ORGANIZACIÓN
}

\section{INCLUSION IN CHILDHOOD EDUCATION IN SPAIN FROM CASES STUDY ON THE SCHOOLS SPACES AND ORGANIZATION}

\author{
Eladio Sebastián-Heredero ${ }^{1}$ \\ Laura Gil, Marina López, Marta Loeches y Lorena López ${ }^{2}$
}

\begin{abstract}
Resumen
El espacio escolar es el lugar donde se organiza el aula ya que es donde trabaja el niño a diario en la escuela, convirtiéndose en un instrumento de convivencia, de educación social, de perfeccionamiento de las relaciones sociales y de inclusión. En este recorte de una investigación mayor vamos a centrarnos en los espacios interiores y exteriores de aulas de educación infantil como lugar donde acontece la acción educativa para todos los alumnos y por tanto un espacio de inclusión. Por ello veremos la organización y ubicación del mobiliario, las actividades a realizar, los materiales y la decoración en sus planteamientos teóricos para pasar a continuación a ver como se organizan en algunos centros educativos.
\end{abstract}

Palabras-clave: inclusión escolar. espacios escolares. organización de aula.

\begin{abstract}
The school environment is the place where the classroom is organized due to is the place where the child works at school daily, becoming in an instrument of connivance, of social education and a improving of the responsibility, considering indoor and outdoor spaces, the organization and the location of the furniture, activities to do, materials and the decoration.
\end{abstract}

Keywords: space. Organization. Classroom. Materials. Furniture.decoration.

\section{INTRODUCCION}

Durante muchos años se buscó siempre un lugar ideal en el que impartir las clases, y en este tiempo fueron muchas las experiencias, desde los peripatéticos al modelo de escuela actual han sido muchas las variaciones, lo que parece evidente, como

\footnotetext{
${ }^{1}$ Profesor-Doctor de la Universidad de Alcalá. eladio.sebastian@ gmail.com

2 Alumnas del Curso de graduación en Magisterio de la UAH (España) 1.gilv@edu.uah.es, marta.loeches@edu.uah.es, marina.lopezg@edu.uah.es, lorena.lopezl@edu.uah.es, C/ Madrid, 119001 Guadalajara, Facultad de Educación de la Universidad de Alcalá.
} 
dice Sebastián (2009, p. 301) es la relación entre los espacios escolares y el clima de la institución y la relación que en ese espacio se establece. Nos corresponde a nosotros como docentes organizar estos espacios para quepan todos, es decir, que sean inclusivos.

Podríamos decir que fue en 1838 cuando se aprueba el Reglamento Provisional de Escuela Publicas donde se definen las características para el espacio asignado para impartir clases y por tanto el modelo de escuela actual.

A principios del siglo $\mathrm{XX}$ surgen diferentes teorías acerca de la arquitectura escolar que crean un compromiso que obliga a construir escuelas. Aparecen diferentes filosofías pedagógicas como las de María Montessori que repercutirá en las construcciones escolares con la creación de escuelas abiertas en contacto con el exterior, pero supuso un fracaso en algunas escuelas; la corriente de Fröebel aborda el diseño de los espacios-escuela fundamentándose en sus conocimientos de cristalografía y la influencia de las tradiciones simbólicas o la Escuela Nueva propone que el alumno debe sentirse integrado en el propio centro, estando en contacto con la naturaleza y que las aulas sean de mayores dimensiones.

En un principio las edificaciones escolares, como dice Sebastián (2008, 20) tenían una arquitectura simétrica y una separación por géneros. A finales del siglo XIX se inicia un proceso de renovación del edificio de la escuela vinculando al arquitecto Antonio Flórez. A principios del siglo XX, Rufino Blanco afirmó que la construcción tiene que estar dotada de una zona central desde la que se pudiesen ver todas las aulas, mediante cristaleras En 1912 la dirección General de Primera Enseñanza consideró que lo más importante es la estética y decoración interior y exterior del centro para que sea diferenciado de otros edificios.

En 1953 se promulgó el primer Plan de Construcciones Escolares y con éste surgen iniciativas; proyecto tipo de escuelas graduadas, I plan nacional de construcciones escolares, construcciones escolares. Un capítulo de los "Pactos de la Moncloa" 1977, dio lugar a un amplio acuerdo sobre los temas de Política Educativa y con carácter significativo a construcciones escolares. La promulgación de la LODE y de la LOGSE supuso un cambio en la modificación de la organización de la enseñanza que afectó a los espacios escolares. 
Pasados los años noventa se construyeron nuevos centros educativos que dieran cabida a la generalización de la enseñanza obligatoria y gratuita hasta los dieciséis años y la escolarización del alumnado de educación infantil. Ello, supuso grandes cambios y reformas: la escolarización de alumnos con necesidades educativas especiales implicaba la necesidad de adaptar espacios, accesos y materiales y se incluyen una serie de servicios a la escuela. Hay una división de la enseñanza pública y la privada. En el siglo XXI, se dio la descentralización de la educación. La promulgación de la LOE (2006) hace que se aborden las construcciones escolares desde otra perspectiva.

La organización de los espacios de los centros, se dice en España (2013), debe estar estructurada y adaptada a las necesidades que puedan surgir en el desarrollo de los niños con un cuidado en la atención a la diversidad, respetando la heterogeneidad que se pueda dar. Consideramos que la organización en el aula es importante para conseguir un ambiente favorable que permita un buen desarrollo de todos los escolares de la misma forma que partimos de la base de que en las escuelas de la muestra en todas sus clases hay alumnos con necesidades educativas especiales. Se deberá tener en cuenta los elementos que conforman el aula como la iluminación, el ruido, los materiales, la accesibilidad, etc. para conseguir unas condiciones óptimas que no influyan negativamente en los niños. Desde esta perspectiva es necesario hacer un especial hincapié en la distinción de los espacios interiores y exteriores, ya que generalmente se da importancia sólo a la organización del aula sin tener en cuenta que la organización del centro también incluye espacios exteriores. Así mismo, hay que tener en cuenta también los equipamientos y materiales ya que será necesario ir adaptándolos al temario y a las diversas situaciones que se presenten, modificándose incluso la organización del aula dependiendo de estas.

\section{ORGANIZACIÓN DEL ESPACIO EN LOS CENTROS EDUCATIVOS}

El espacio en los centros educativos debe ser un ambiente idóneo en el que se sientan a gusto los alumnos y el profesorado, como dice Duarte (2003) y se aproveche al máximo el material docente que se posee, intentando incrementar la motivación de 
los niños por aprender, explorar, investigar y descubrir, ampliando las experiencias, enriqueciendo la dotación de recursos y materiales pedagógicos con elementos que favorezcan la integración de los aspectos cognitivos, motores, sociales, emocionales, comunicativos e interactivos, etc.; es el lugar donde se dan los aprendizajes y el propio espacio es lugar de aprendizaje como dice Sebastián (2008).

En estos espacios los niños van a experimentar y a empezar a formarse como personas es donde debe acontecer la inclusión. Dentro de la organización de los espacios hay que cuidar tanto el fondo como la forma para que puedan ser espacios educativos cargados de significado y de contenido para todos los alumnos y alumnas y precisamente las aulas de educación infantil nos pueden enseñar mucho.

\subsection{Organización del espacio interior}

Consideramos el aula como espacio interior por definición, destinada al trabajo diario, donde lo más importante es la organización de la misma y en el cómo colocar el mobiliario y la distribución del material aprovechando todo el espacio, que va a hacer que dos aulas similares, realmente sean muy diferentes, como vemos en Pontiveros (2011). Para que el espacio sea entendido como algo propio, en la distribución de los materiales de clase pueden participar los niños lo que hará más atractiva y participativa la clase colocándolos según sus intereses. Es importante, también, que las familias colaboren para la mejora de las instalaciones del centro.

Es importante que la organización del espacio facilite el encuentro entre los miembros del grupo, a la vez que permita el aislamiento, el trabajo y el juego individual, la autonomía personal derivada de sus características personales.

En la actividad colectiva desarrollamos actividades de pequeño grupo, con su equipo. (la elaboración de murales y disfraces etc.); actividades de gran grupo, con toda la clase (excursiones, juego de patio...) y actividades conjuntas con la clase paralela, (celebraciones festivas...) lo que significa adoptar un modelo de distribución del espacio que ya en si mismo nos indica modelos inclusivos o de exclusión.

En la actividad individual el trabajo personalizado es el preferente y para ello depende de la organización y facilitación de distintos materiales. En educación infantil 
organizar la clase por rincones es una buena manera de que, según sus necesidades, jueguen y aprendan espontáneamente potenciando la autonomía y todos puedan desarrollarse. Esto recoge dinamismo, plasticidad y flexibilidad. Se permite que los niños escojan las actividades que quieren realizar, dentro de unos límites. Fomentando el maestro el interés y la curiosidad en el aula. Los rincones dependerán de la capacidad organizativa del maestro/a y del espacio de que disponga, pero si esta contrastada su eficacia por qué no utilizarla en otras etapas educativas.

Además del mobiliario las paredes del aula tienen una importante función ya que constituyen un medio de aprendizaje y estímulo al poder fijar en ellas los trabajos realizados por los niños, puede ser el lugar donde los alumnos vean sus realizaciones.

Los espacios interiores también engloban: la entrada, que es el primer espacio de toma de contacto con el centro; los servicios, es donde se realizan aprendizajes relacionados con hábitos de higiene, orden y convivencia; las salas de usos múltiples, como complemento y que puede ser utilizada como taller, sala de psicomotricidad, sala de juegos... los espacios de circulación y comunicación: referidos a los pasillos y las escaleras que permiten la circulación física de las personas; las zonas gestión: la secretaría, conserjería, dirección y sala de profesores y también otras salas de reunión y de trabajo;

La importancia de la accesibilidad queda fuera de toda duda, como dice Solorzano (2013) y tanto los centros como las administraciones educativas han de trabajar por ello en pro de la igualdad, la realidad que nos encontramos es que de forma general esta se da en los centros analizados.

\subsection{La organización del espacio exterior}

El proceso de enseñanza-aprendizaje no se limita al aula, todos los espacios tienen un potencial educador que debe ser aprovechado en la práctica pedagógica cotidiana.

La organización de los espacios exteriores hace referencia a los espacios abiertos, como el patio de recreo, lugares donde los que los niños pueden poner de 
manifiesto su iniciativa y su creatividad participando con una mayor libertad dice Pontiveros (2011), tanto individuales como en grupo. El patio de recreo y zonas deportivas debe entenderse como un gran espacio común, lugar de reunión de todo el centro, donde además de juegos dirigidos o libres poder realizar otras actividades en común;

Las ventajas del uso del espacio exterior son múltiples, y sus limitaciones escasas. Deberán estimular el movimiento y destrezas motóricas; espacios libres y amplios para poder correr y desplazarse libremente; ser un espacio amplio y de fácil acceso desde las aulas, con un suelo variado, un espacio al sol y a la sombra, además de un espacio cubierto para épocas de lluvia. En él también podemos incluir toboganes, columpios, neumáticos y otros rincones para la relajación y concentración individual, como jardines, huertos, etc. lo que les dotará de significado y de contenido. Analizar los espacios naturales que puedan existir o crearse e integrarlos en el espacio lúdico puede ser un gran proyecto de inclusión.

\section{EQUIPAMIENTOS Y MATERIALES}

Entendidos como el conjunto de elementos complementarios a la estructura básica del edificio y sus dependencias que facilitan la tarea docente. Los equipamientos deben permitir la movilidad del alumno potenciando la autonomía y integración de todos los alumnos.

Otros aspectos del aula como la iluminación favoreciendo la natural ya que facilita las tareas visuales del alumno, evitando el deslumbramiento en el aula; una buena ventilación y la ausencia, o reducción, de ruido para que no interfiera en las tareas intelectuales. En algunos casos de alumnos con necesidades educativas especiales estos aspectos son de importancia muy significativa.

Respecto a los materiales que se pueden utilizar en el aula los más relevantes serán: los materiales para el desarrollo motórico y sensorial, el material didáctico, materiales de manipulación, observación y experimentación, materiales que desarrollan el pensamiento lógico, materiales para la representación y simulación y materiales para el desarrollo de la comunicación oral, que como vemos están directamente relacionados 
con la atención a la diversidad.

\section{LA ORGANIZACIÓN DEL ESPACIO FÍSICO EN ALGUNOS CENTROS ESCOLARES}

Para este análisis tomamos las ideas que nos aportan Iglesias (2008); Laguía y Vidal (2010) y Sebastián (2008) de cara poder tener un referente claro en nuestra mirada y reflexión sobre los espacios educativos en general y los de educación infantil en particular con sus singularidades.

3.1 Organización física del aula: Decoración, material, metodología,... en el Colegio no 1 de la muestra

La organización del espacio en este centro privado se realiza por zonas con accesibilidad total a espacio y materiales. Existe la zona de la alfombra (zona más importante) que es donde se realiza la asamblea, la zona de juegos y la zona de la pizarra digital, aunque nunca dejando de lado la pizarra tradicional ya que es un buen instrumento para trabajar la lecto-escritura.

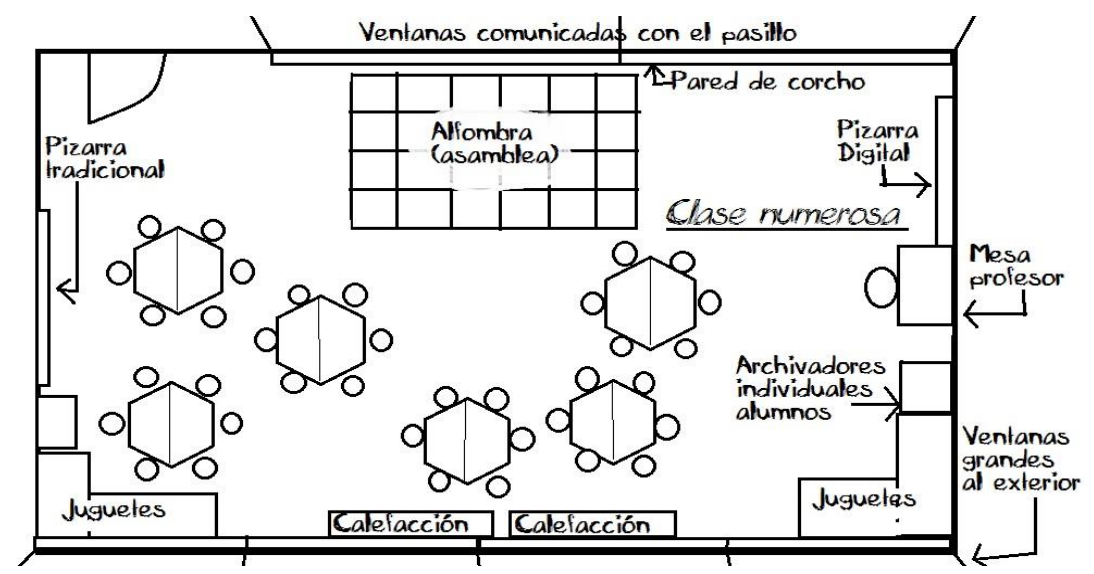

No se trabaja por rincones y se realiza un trabajo de estimulación temprana a través de un circuito por el resto de las instalaciones del centro. Cada clase consta de un espejo y un ordenador, y las mesas están cerca de las ventanas, aunque hay aulas en las que las mesas no se podían situar en esa posición debido a la ubicación de la pizarra. 

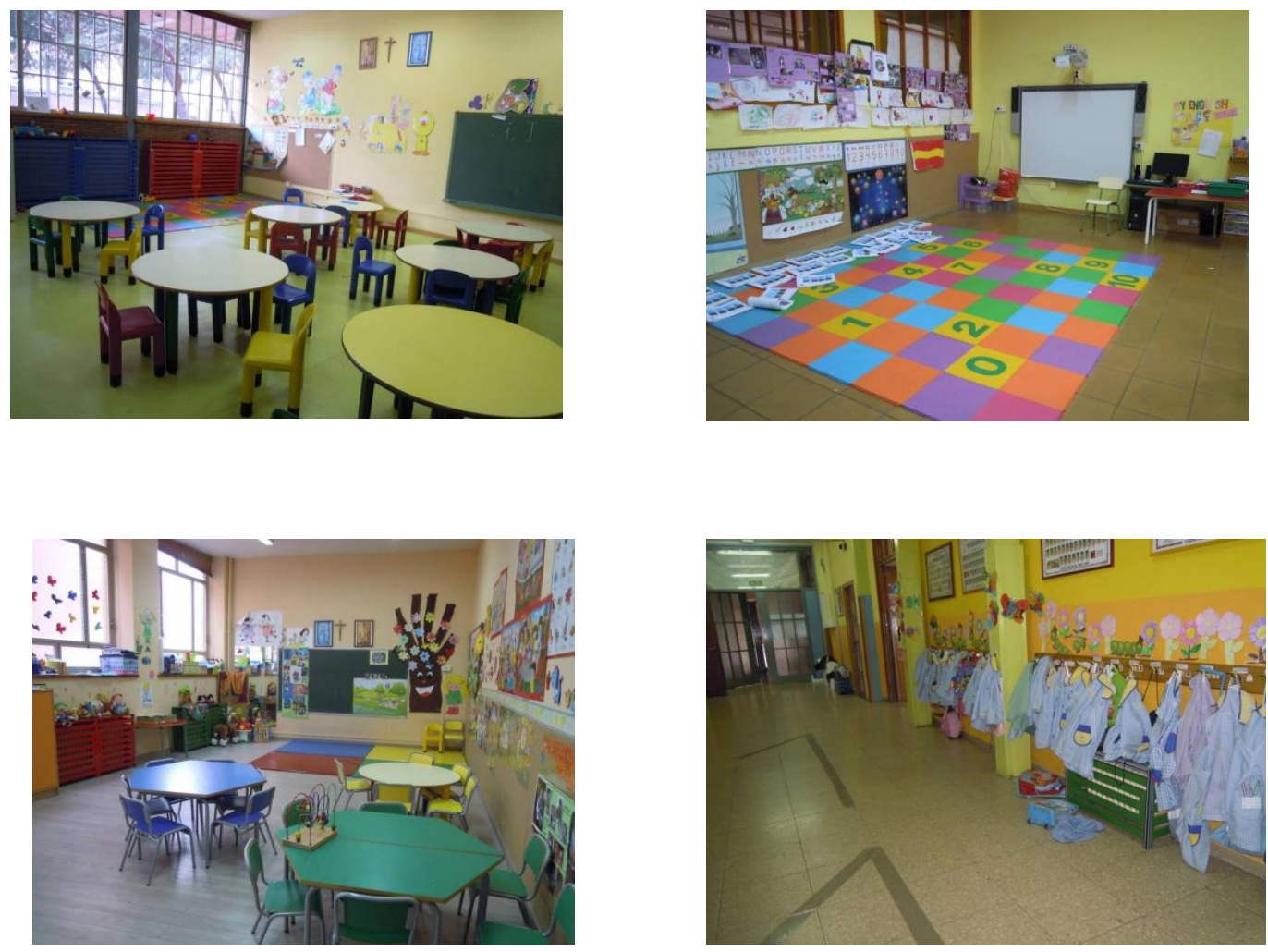

Las aulas están decoradas con los trabajos realizados por los niños y se va cambiando cada poco tiempo, también se cambia la decoración de los pasillos de acuerdo con la temática que se esté dando.

Los materiales que se utilizan en las aulas son los juegos, que se realizan en la alfombra, las fichas, que se realizan en las mesas, y en la pizarra digital los trazos. Cada alumno tiene un material propio comprado por los padres (lápices, gomas...) pero el resto de los materiales se los proporciona el centro (cartulinas...).

El centro no consta de un patio adaptado a las necesidades de los niños. Pero sí tiene una zona vallada para niños de 2 años.

La profesora está cómoda con la organización del aula y los alumnos también, fue ella misma la que organizó la decoración y la disposición en medida de lo posible, aunque había algunos inconvenientes a la hora de colocar las mesas ya que deben estar colocadas cerca de la pizarra y en algunos casos la pizarra no estaba cerca de la ventana debido a que el edificio conserva las mismas aulas desde el día de su construcción e incluso algunas aulas se sitúan en dependencias con otras finalidades en antaño. 
También nos comenta que le gustaría disponer de más pizarras digitales ya que al tener pocas, los diferentes grupos tienen que compartirla y acudir todos a la misma aula.

\subsection{Organización física del aula: Decoración, material, metodología,... en el}

\section{Colegio $n^{0} 2$ de la muestra}

Respecto a la organización del aula, en este centro privado, cabe destacar que en el primer ciclo trabajan por fichas y en el segundo ciclo por rincones y a su vez trabajan por bits. La clase está distribuida en grupos de mesas según el rincón o ficha y el de arte está siempre al lado del baño o lavabo. Tanto la pizarra digital como la tradicional se encuentran ubicadas en un lugar visible para toda la clase. Los materiales están al alcance de los niños y hay un espacio destinado a la asamblea. Podriamos decir que la accesibilidad de espacio y materiales es total. Los profesores realizan pequeñas modificaciones de clase según su preferencia. El colegio tienen una "peque-teca" que es un espacio exclusivo en el cual los niños realizan actividades con sus libros y un gimnasio.

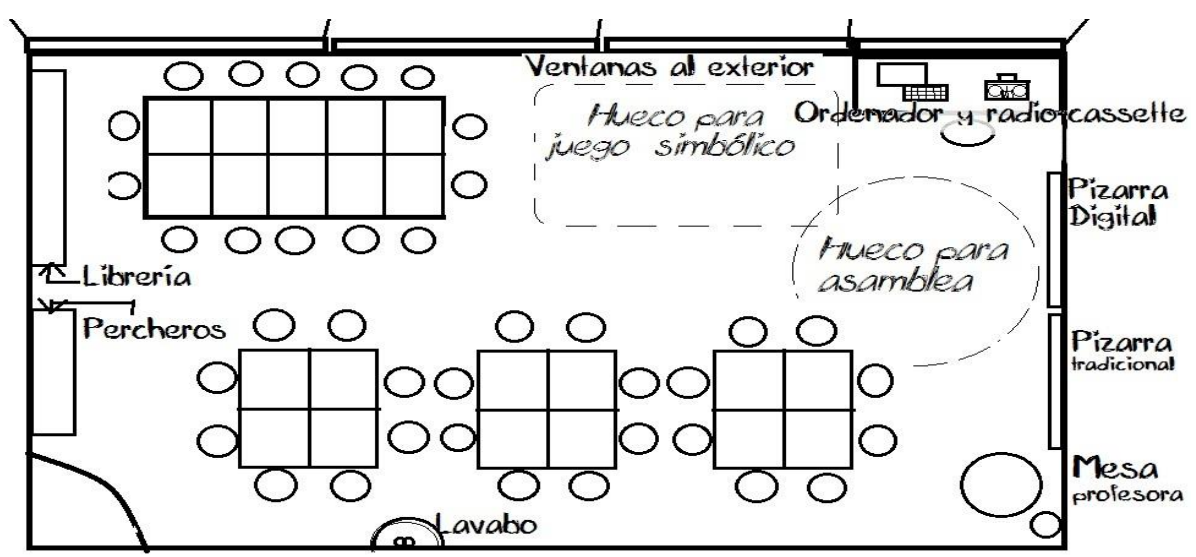

La organización de los grupos en el aula se basa en el trabajo por rincones, por lo que la profesora procura sentar a niños que presentan más dificultades con los que no las tienen para que éstos les estimulen a los primeros, adaptaciones no significativas que dan buenos resultados en alumnos co, problemas de aprendizaje. En el aula se realizan apoyos de lectura y lógica-matemática para los alumnos con nee y/o deficiencias. Toda la organización de tiempo se rige por un horario establecido. Cuando los niños tienen 
lengua, inglés y música, se imparte la clase en el aula pero psicomotricidad lo dan en el gimnasio y piscina.

Los pasillos son amplios para que cuando no puedan salir al patio por ocasiones de lluvia puedan liberarse en él. A la vez los ventanales son grandes para evitar el empleo de luz artificial y las puertas y las ventanas cuentan con sistemas de seguridad. Además el primer ciclo tiene calefacción que va por el suelo y en el segundo ciclo radiadores.

A lo largo del curso la decoración del aula va cambiando según las unidades o proyectos que realicen tanto en inglés, lengua, reciclaje ecológico o arte. Encontramos murales, calendarios, setas donde se apunta quien va cada día a clase, fotos, dibujos, abecedario, cumpleaños. Cuentan con un árbol que según la estación del año en la que estén lo decoran.

Todos cuentan con el material habitual propio que se encuentra en los archivadores de cada uno, en cada rincón encontramos diferente materiales/juegos, para actividades libres y dar espacio a los diferentes ritmos de aprendizaje. Cuentan con un ordenador, pizarra tradicional y digital. Se da la presencia de colchonetas para que los niños se echen la siesta hasta 3 años. Cada uno tiene su taza que está colgada alrededor del lavabo. Los padres se implican también a la hora de traer el material a clase.

Encontramos dos espacios exteriores, para el primer ciclo cuentan con un patio que tiene conexión directa a través de las clases. Este patio cuenta con suelo blando de goma, corre pies y toboganes. En el segundo ciclo encontramos que tienen un arenero, toboganes y un laberinto de sensaciones.
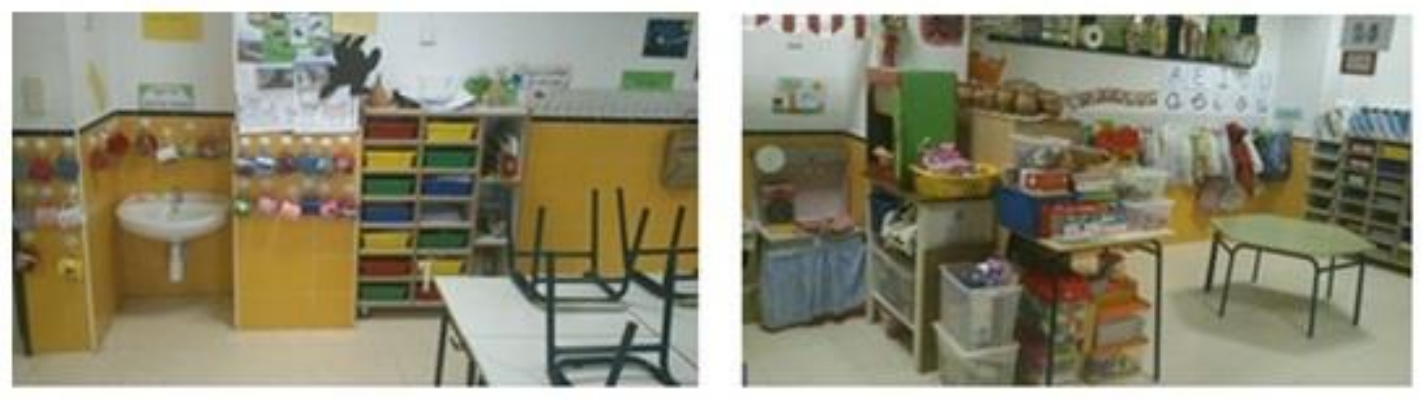

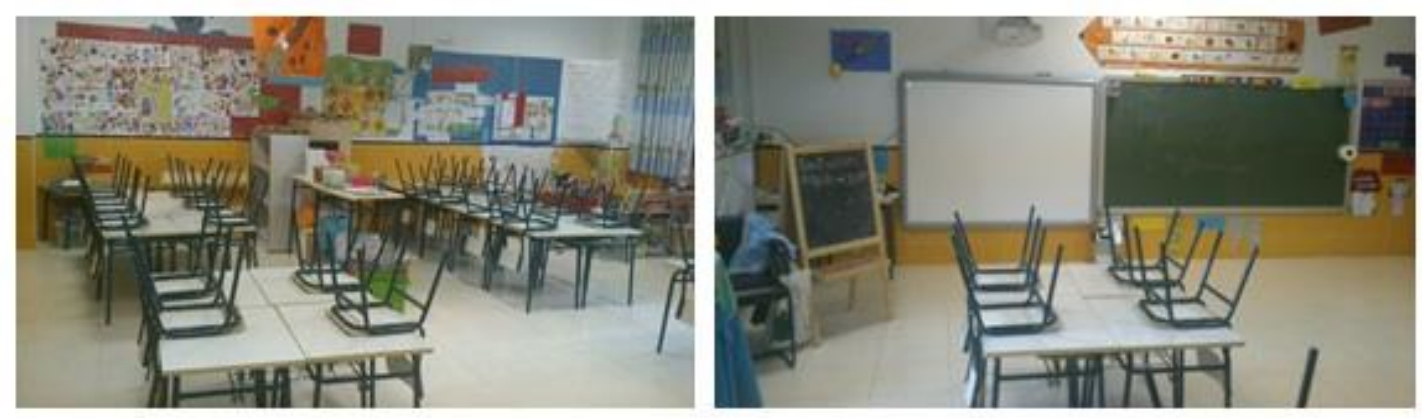

Los profesores están muy contentos al trabajar por rincones en el segundo ciclo, ya que lo ve positivo para ellos y motivador. Están entusiasmados ya que han conseguido la peque teca, un taller de alfarería y que vayan al laboratorio ya que da mucha importancia a la experimentación. Por ello quieren añadir también un rincón de ciencias/experiencias. A su vez encontramos a los profesores a favor de la utilización de las nuevas tecnologías como son la pizarra digital o los ordenadores.

\subsection{Organización física del aula: Decoración, material, metodología,... en el Colegio $n^{0} 3$ de la muestra}

En este colegio público, respecto a la organización podemos decir que se trabaja por rincones todo el ciclo. Los profesores tienen total libertad y autonomía en la distribución de la clase y suelen ir realizando los cambios sobre la marcha, aunque es cierto que existen muchos elementos que no permiten esta libertad de movimiento como puede ser la pizarra tradicional, algunos de los armarios, etc. eso hace que exista una accesibilidad total en espacios y a materiales. Además, serán necesarias algunas modificaciones en función del tipo de alumnado, teniendo en cuenta las circunstancias de una niña en silla de ruedas con presencia de discapacidades, evitando que no se quede aislada o ajena a las actividades, y una silla que se adapte a sus necesidades a la hora de trabajar en las mesas junto al resto de sus compañeros o situar a los niños con dificultades en el idioma junto a otros niños que si hablen y que puedan servir de estímulo y ayuda aquellos que les cueste más, de tal forma que los niños se estimulen unos a otros y se favorezca a la integración. 


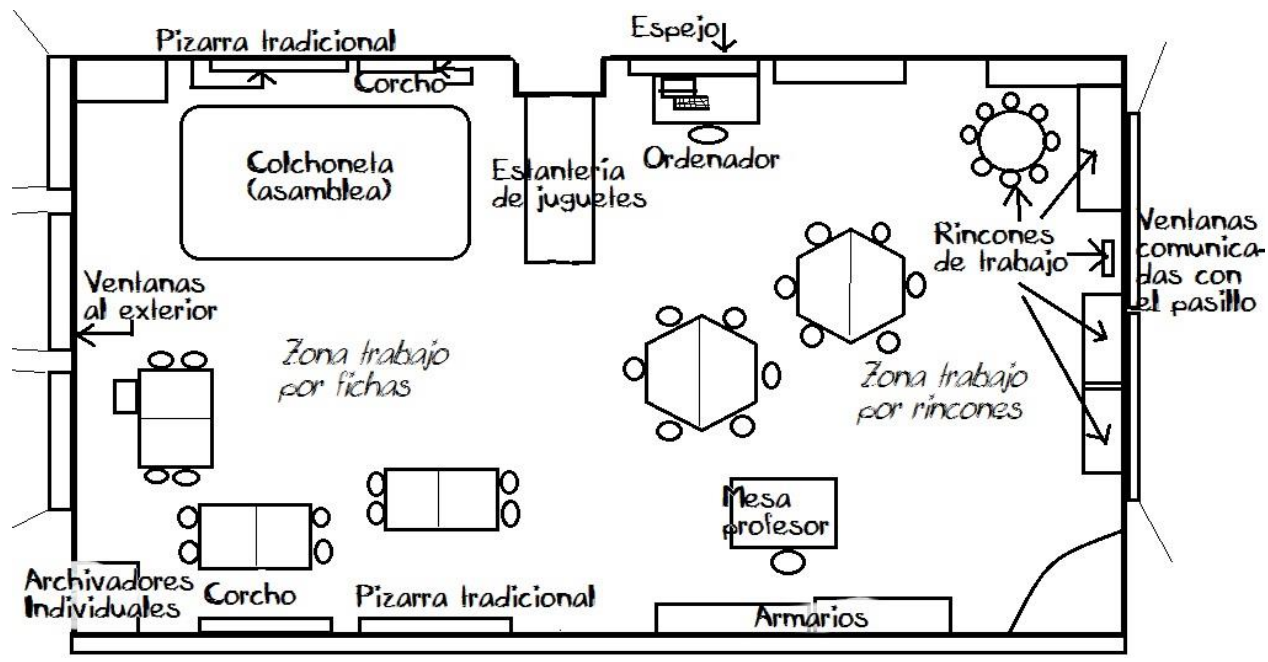

Disponen de pasillos espaciosos con presencia de bancos. Respecto a las ventanas la iluminación natural es muy buena existiendo ambos lados de las clases. Unas que dan al pasillo y otros que dan al patio.

Se dan cambios en la decoración, la cual va cambiando según la unidad que se esté dando, situando los dibujos y trabajos de los niños por las paredes, los corchos,los pasillos y el resto de colegio.

Los mayoría de los materiales que se utilizan los proporciona el centro, aunque los padres si deben encargarse del material básico como lápices, goma, etc. Encontraremos diferentes materiales según el rincón o los juegos que se realicen. A diferencia de los colegios anteriores, tan sólo disponen de pizarras tradicionales de manera que no cuentan con la ayuda que suponen la utilización de una pizarra digital.

Disponen de un patio que cuenta con un arenero, un tobogán, columpios, cuerdas para trepar, etc. Además, cuentan con baños totalmente diferenciados adaptados y separados y diferenciados de los de primaria.
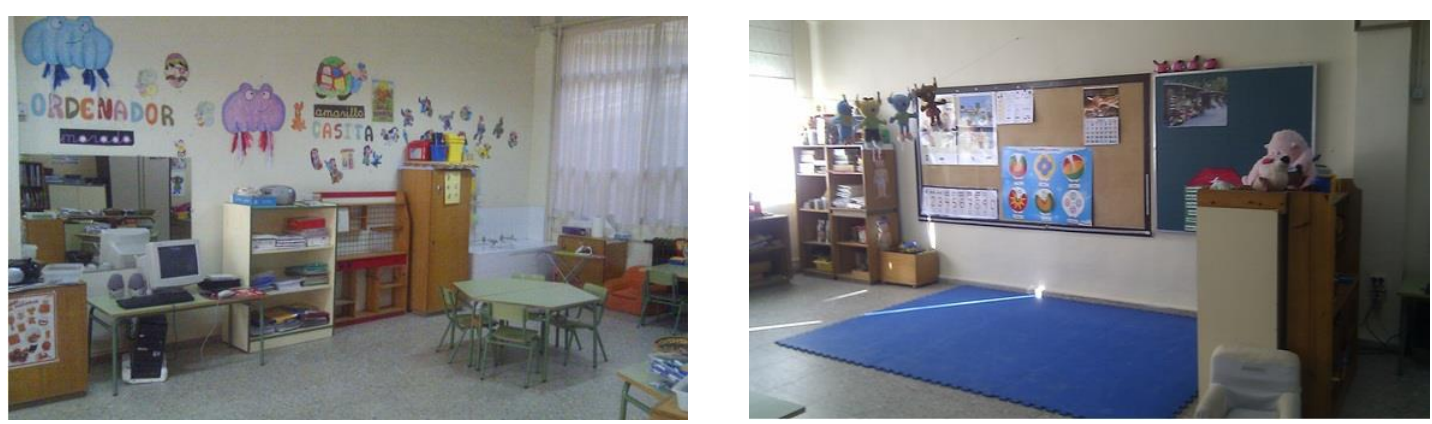

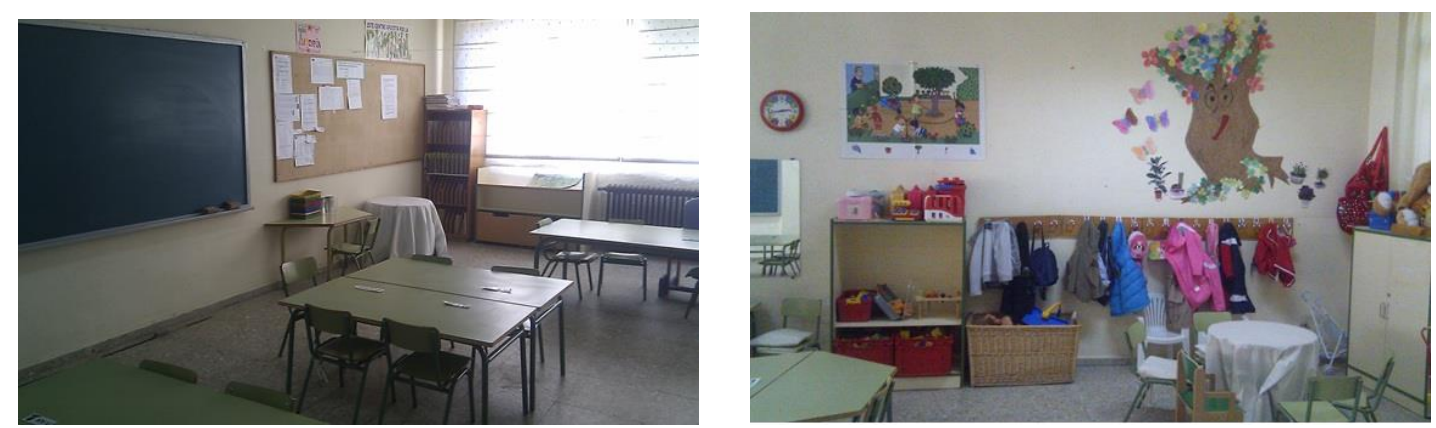

Según nos contaba la profesora, al ser tutores de la clase pueden elegir libremente la organización de la clase para evitar la monotonía, lo que permite trabajar más a gusto al poder ser ellos mismos quienes establezcan la organización en función de las necesidades de los alumnos. El aula se va modificando continuamente. Es cierto, que le gustaría disponer de más materiales manipulables para los niños y más recursos que puedan facilitar su desarrollo, aunque las circunstancias económicas actuales dificultan un poco este deseo. Además, ésta docente afirma que le gustaría cambiar la ubicación de muchas de las pizarras pero debido a la existencia de un anclaje al panel de madera que comunica un aula con la otra, es imposible. A diferencia de esta profesora, nos encontramos con otro profesor que tiene preferencia por las fichas y considera los rincones como método de juego.

\section{CONSIDERACIONES FINALES (conclusión y futuro)}

Después de lo visto hasta aquí, podemos observar como en estas tres muestras encontramos una estructura básica buena, aulas amplias y diversas, espacios adecuados y con riqueza de materiales comunes y accesible a todos los alumnos. Dentro de esto observamos la existencia de espacios dotados de contenidos diversos y muy amplios y modificables, lo que les dota, a priori, de características inclusivas.

En cuanto a los patios del colegio, hemos apreciado una notable diferencia ya que en dos de ellos, el patio no se adapta plenamente a las características de los niños incluidos los que tienen necesidades educativas especiales y/o deficiencias. A diferencia de esto, el colegio $\mathrm{n}^{\mathrm{o}} 2$, tiene diferentes espacios en función de las edades, con diferentes tipos de suelo y juegos, lo que permite una gran versatilidad y uso 
diferenciado, estas configuraciones los hace espacios inclusivos y de diversidad.

En todos los centros dan importancia a la estructura de la clase teniendo en cuenta la iluminación pero sobre todo la circulación, considerando que en un caso hay una alumna con silla de ruedas, lo que significa que se trabaja la accesibilidad, por tanto no basta con tener el recurso, hay que adaptarlo a las circunstancias hecho que aquí aparece.

La organización de los rincones la hemos visto en dos centros esto permite trabajar distintas actividades de forma simultánea, es una forma clara de poder atender a las singularidades y necesidades de cada alumno, y una respeto a los ritmos del alumno. También pudimos observar que dentro de un mismo colegio los profesores pueden trabajar con diferentes metodologías, para atender las necesidades educativas y dar respuesta de forma más adecuada, empleando los rincones como complemento al trabajo por fichas y no como una actividad en sí misma. Los rincones permiten trabajar por grupos pequeños y que los niños se socialicen mejor y además se fomenten el desarrollo de las diferentes inteligencias múltiples.

Respecto a la decoración en la muestra analizada se basa, para trabajar, en un tema en común respecto a las unidades que se trabajan, y se va modificando a lo largo del curso, un recurso que se considera importante en atención a la diversidad. Consideramos, a tenor de lo expuesto, que las familias deberían implicarse más en la educación de sus hijos, en el momento de realizar un mural, aporte de material, colaboración en actividades del colegio...

Destacamos la incorporación de las pizarras digitales y su papel en las nuevas aulas para cercar los recursos de internet y materiales lo que sin duda puede ser de gran utilidad para los alumnos con necesidades educativas.

A partir de aquí consideramos que las posibles mejoras de la educación no sólo dependen de la actividad de los docentes sino que también sería importante concienciar a la sociedad de la importancia de la educación y de la inclusión.

De otro lado, defendemos, a la vista de lo expuesto, una modificación de la organización de las aulas, con una mayor aportación de materiales y variedad de estos que logre una enseñanza con igualdad de oportunidades (equidad) y que desarrolle otros tipos de metodologías dando más importancia a otras áreas que anteriormente no han 
tenido en cuenta en el desarrollo del niño, recursos de mayor calidad, mayor variedad de aulas, etc.

Además, la gran importancia que están alcanzando las nuevas tecnologías actualmente implica la necesidad de introducir este recurso en el aula, ya que va a ser algo que van a utilizar en su vida cotidiana y como medio de atención a la diversidad. Es importante destacar que el empleo de las nuevas tecnologías es algo que va a repercutir en la organización del aula ya que las actividades que se realicen van a ser complementados con este recurso y las metodologías totalmente diferentes.

Como instrumento didáctico, el espacio está al servicio de la didáctica. Aunque choca con el contrasentido de que pocos espacios son tan poco aprovechados como las escuelas. De cualquier manera habría que reflexionar sobre la apertura del espacio escolar al medio. Por otro lado la metodología exige una flexibilidad espacial para adaptar el lugar físico donde ésta se tiene que aplicar a las exigencias que la diversidad precise en cada caso.

En la escuela como lugar de convivencia y de relaciones algunos espacios se configuran para el trabajo, pero es dentro de este espacio de trabajo donde se articulan las principales relaciones del alumnado y la verdadera inclusión. No podemos olvidar por otro lado que hay un contenido curricular que es enseñar a convivir y que una de las capacidades básicas es la relación interpersonal. El buscar en los centros educativos que sean espacios de convivencia más libre y en el que las normas y reglas sean compartidas.

En conclusión, pensamos que los colegios deben trabajar por conseguir una formación integral de los escolares permitiendo un desarrollo completo en el que participen activamente todos los agentes que forman parte de la educación y el espacio también enseña, es un espacio de inclusión.

\section{REFERÊNCIAS}

DUARTE, J. Ambientes de aprendizaje. Una aproximación conceptual. Estudios Pedagógicos, n. 29, Valdivia, p.97-113, 2003, Disponible en: <http://dialnet.unirioja.es/servlet/busquedates?t=LA+ORGANIZACI\%C3\%93N+DE+L 
OS+ESPACIOS+Y+DEL+TIEMPO\&db=1\&td=TES\&fi=\&ff=\&idi=0>. Tomado el: 3 maio 2015.

ESPAÑA. Unidad 5. La organización de los espacios educativos. Madrid: MINISTERIO DE EDUCACIÓN. 2013. Disponible en: <http://www.minedu.gob.pe/dinfocad/modernizacion/Unidad05.pdf> Tomado el: 3 maio2015.

IGLESIAS, M. L. Observación y evaluación del ambiente de aprendizaje en educación infantil: dimensiones y variables a considerar, Revista Iberoamericana de Educación, $\mathrm{n}^{\mathrm{o}}$ 47, p. 49-70, 2008. Disponible en: 〈http://www.rieoei.org/rie47a03.pdf>. Tomado el: 2 maio 2015.

LAGUÍA, M.J. y VIDAL, C. Rincones de actividad en la escuela infantil de 0-6 años. Barcelona: Ed Grao, 2010.

PONTIVEROS, R. La organización de los espacios y del tiempo. Criterios para una adecuada distribución y organización espacial y temporal. Ritmos y rutinas cotidianas. La evaluación de los espacios y del tiempo. Innovación y Experiencias Educativas, $\mathrm{n}$ 38, Enero, Granada, pp. 1-12, 2011. Disponible en <http://www.csicsif.es/andalucia/modules/mod_ense/revista/pdf/Numero_38/ROCIO_PONTIVEROS_1 .pdf>. Tomado el 2/5/2015.

SEBASTIÁN, E. A la calidad de la educación desde los espacios escolares: visión del profesorado y perspectivas en la educación castellano-manchega. Universidad Alcalá de Henares (UAH). 2008.

SEBASTIAN, E. Relación entre los espacios escolares y la convivencia. In:

SEBASTIAN, E. y MARTIN, M. Formación del ciudadano global. Alcalá: Serv Publicaciones UAH, 2009. p. 3013-318.

SOLÓRZANO, M. J.Espacios accesibles en la escuela. Revista Electrónica Educare. 2013. Disponible e : <http://www.scielo.sa.cr/scielo.php?pid=S140942582013000100006\&script=sci_arttext>. Tomado el: 2 maio 2015. 